\title{
Valorization of Waste Chicken Feathers: Fabrication and Characterization of Novel Keratin Nanofiber Conduits for Potential Application in Peripheral Nerve Regeneration
}

\author{
Mduduzi Khumalo $\mathbb{D}^{1,}{ }^{1,2}$ Bruce Sithole, ${ }^{1,2}$ Tamrat Tesfaye $\mathbb{D}^{1,2,3}$ and Prabashni Lekha ${ }^{2}$ \\ ${ }^{1}$ University of KwaZulu-Natal, Discipline of Chemical Engineering, Durban, South Africa \\ ${ }^{2}$ Biorefinery Industry Development Facility, Chemical Cluster, Council for Scientific and Industrial Research, Durban, South Africa \\ ${ }^{3}$ Ethiopian Institute of Textile and Fashion Technology, Bahir Dar University, Bahir Dar, Ethiopia
}

Correspondence should be addressed to Mduduzi Khumalo; mdukhumalo@hotmail.com

Received 8 June 2021; Revised 13 December 2021; Accepted 8 January 2022; Published 28 January 2022

Academic Editor: Xiaoming Li

Copyright (c) 2022 Mduduzi Khumalo et al. This is an open access article distributed under the Creative Commons Attribution License, which permits unrestricted use, distribution, and reproduction in any medium, provided the original work is properly cited.

\begin{abstract}
Flexible, porous, biocompatible, and biodegradable tubular keratin nanofibers were fabricated as nerve regeneration conduits. Keratin was extracted from waste chicken feathers and then blended with polyvinyl alcohol and transformed into nanofiber conduits by electrospinning. The nanofiber conduits had average diameters that ranged from 170 to $234 \mathrm{~nm}$. The nanofibers' average diameter decreased when the keratin content was increased. In contrast, the range of nanofiber diameter distribution narrowed, suggesting that as nanofibers became thin, their numbers increased, thus reducing the interfacial spaces between them. The analysis confirmed the presence of keratin protein in nanofibers, guaranteeing biocompatibility and biodegradation. TGA showed that keratin improved the thermal stability and hydrophilicity of the nanofibers.
\end{abstract}

\section{Introduction}

The peripheral nervous system (PNS) is an integral part of the nervous system that enables the body to move muscles and feel normal sensations. Its primary function is to connect the central nervous system (CNS) to tissues and organs [1]. The role of PNS is made possible by central nervous system basic units, called neurons, which are composed of, among others, the bundles of axons that form electriccable-like peripheral nerves. Unlike CNS protected by the skull and vertebrae, the PNS is exposed to injuries that can either be neuropraxia, axonotmesis, or neurotmesis, where neuropraxia is the least severe, while neurotmesis is the most severe nerve injury [2]. The complicated anatomy of the nerve bundle makes nerve repair difficult, leading to the most unsuccessful treatments. Peripheral nerve injuries remain a challenge for both clinically and basic research despite the advancements that have been made in this field [3]. Current repair methods include end-to-end repair, grafts, and synthetic conduits. While end-to-end suturing, extending, and reconnecting the distal stump to the proximal stump are the simplest and preferred nerve repair method, its success is limited to the gap size and the proximal nerve's availability stump. Furthermore, it is also limited to the amount of tension induced during stump extension over a nerve gap; if it exceeds the optimal level, the repair process results in partial nerve recovery. The treatment may be done by donating the nerve from the other part of the body, autograft. The autograft is advantageous in eliminating or minimizing immunological rejection and is considered the reference standard for closing the nerve gap [4]. However, the balance of functionality between the donor and recipient tissue or organ must be established. This balance limits the treatment if there is a substantial loss of nervous tissue that may require multiple small grafts. Another form of nerve donation treatment is an allograft, whereby the nerve tissue is harvested from the donor of the same species. Even though this process increases nerve tissue availability, it also introduces the chances of disease transmission and immunological response $[1,5]$. 


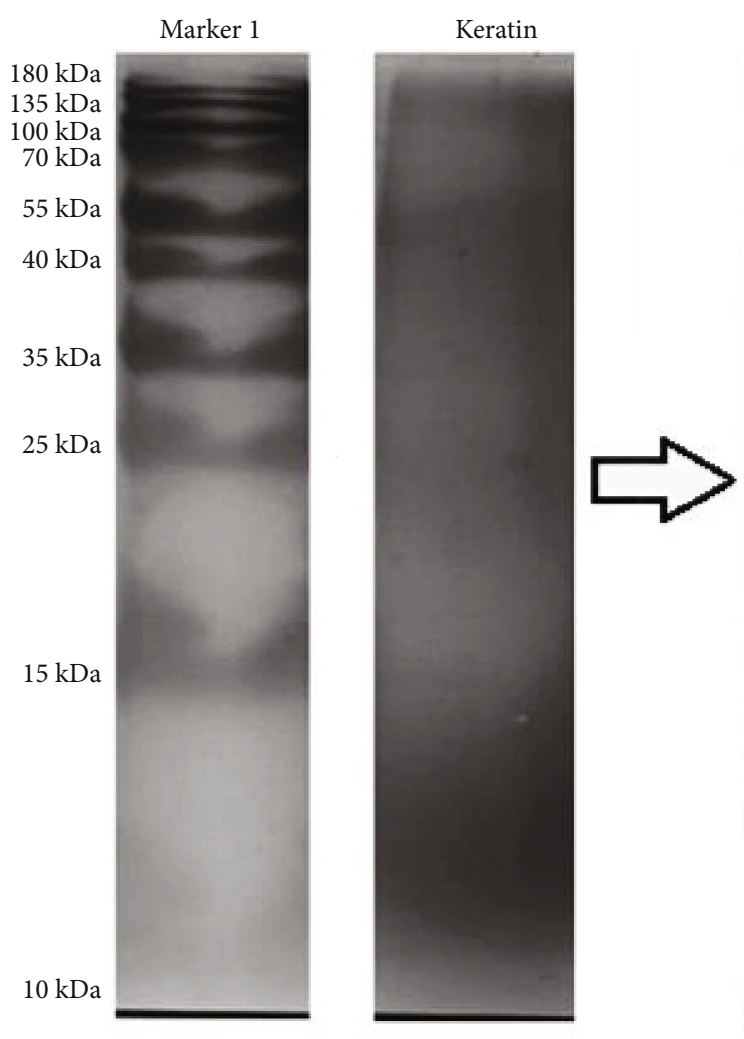

(a)

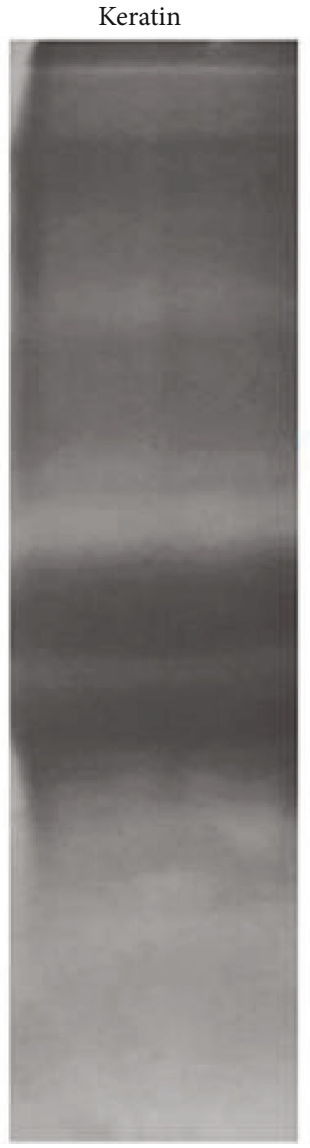

$40 \mathrm{kDa}$

$25 \mathrm{kDa}$

$15 \mathrm{kDa}$

$10 \mathrm{kDa}$

$4.6 \mathrm{kDa}$

$1.7 \mathrm{kDa}$

(b)

FIGURE 1: SDS-PAGE gels of extracted keratin protein: glycine gel (a) and tricine gel (b).

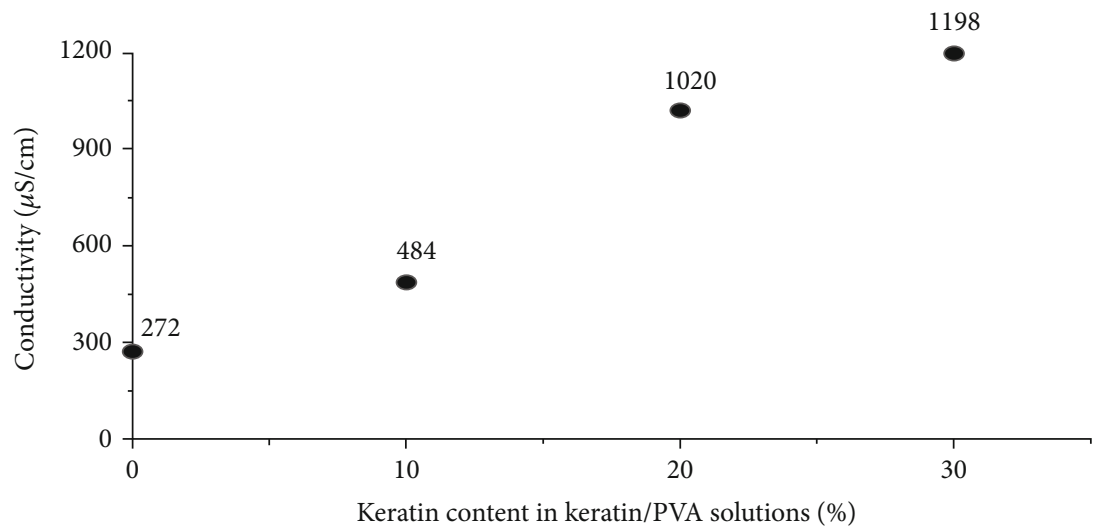

FIgURE 2: Conductivity of keratin/PVA solutions of different keratin and polyvinyl alcohol ratios.

Therefore, synthetic nerve conduits are favoured for the nerve regeneration process because of their flexibility in mimicking the natural nervous tissue. However, the current commercially available conduits are rigid and inflexible and may cause cell loss due to their lack of physiological properties required during nervous tissue movement [6]. The conduit must be biocompatible, biodegradable, porous, bioresorbable, and mechanically strong [7].
Biocompatible synthetic polymers suit most nerve conduits' fundamental requirements because of their adequate mechanical strength, ductility, and physiochemical compatibility, especially electrospun nanofiber scaffolds [8-10]. Nanofibers have attracted attention for their application as nerve conduits due to their softness, flexibility, high porosity, high surface area to volume ratio, and nanoscale diameter $[11,12]$. Research has shown that nanofiber scaffolds 


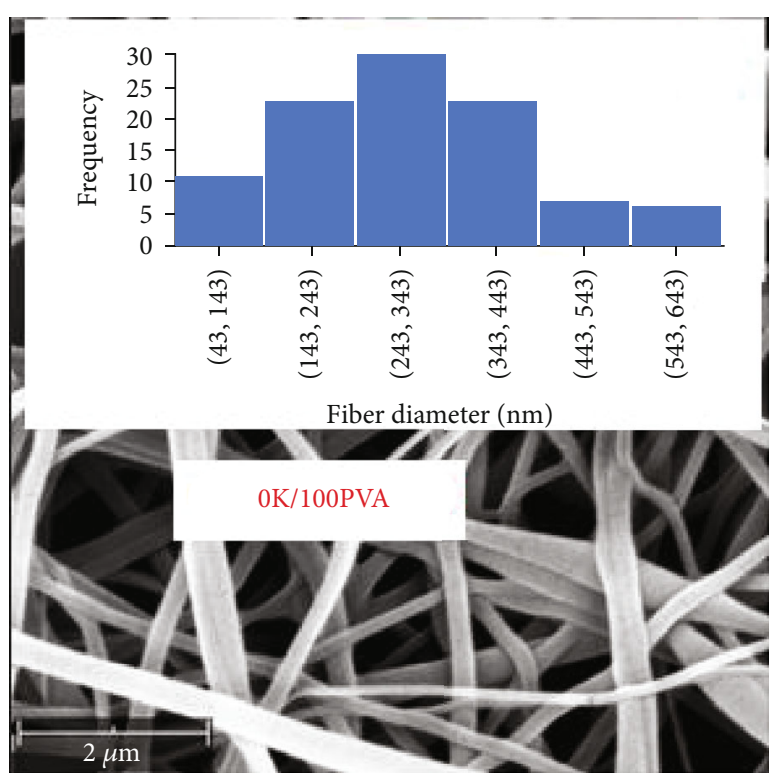

(a)

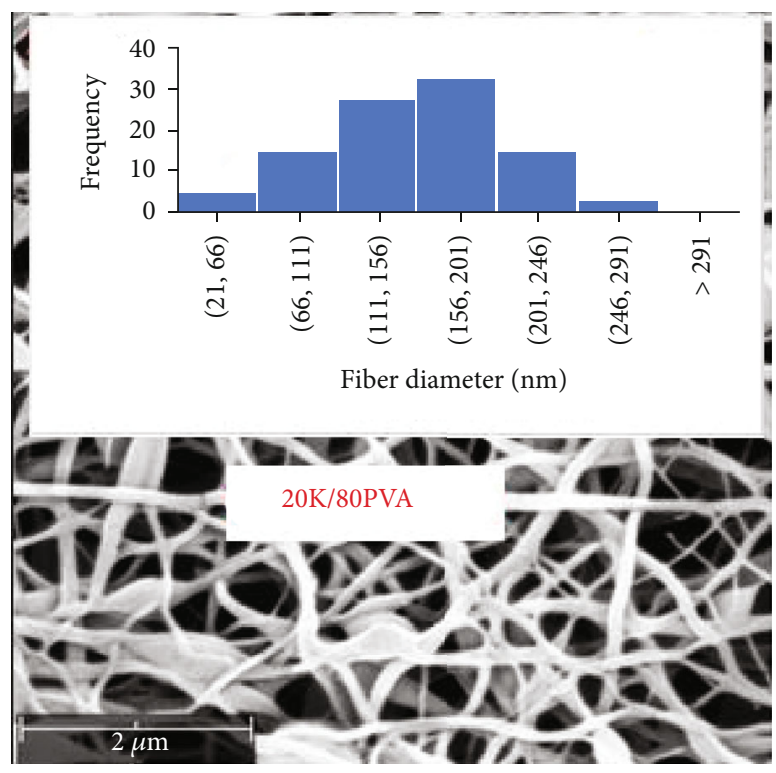

(c)

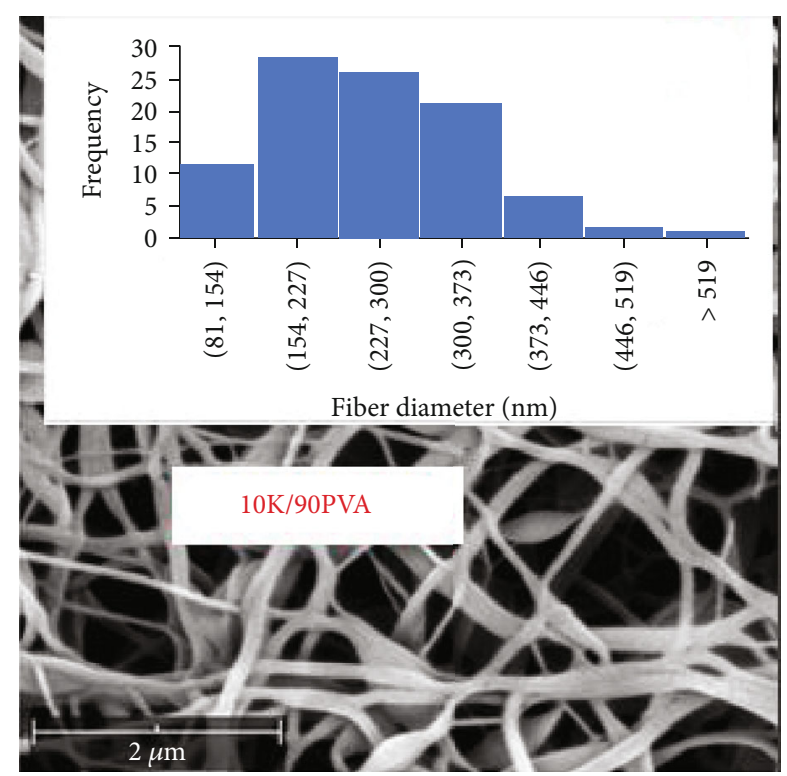

(b)

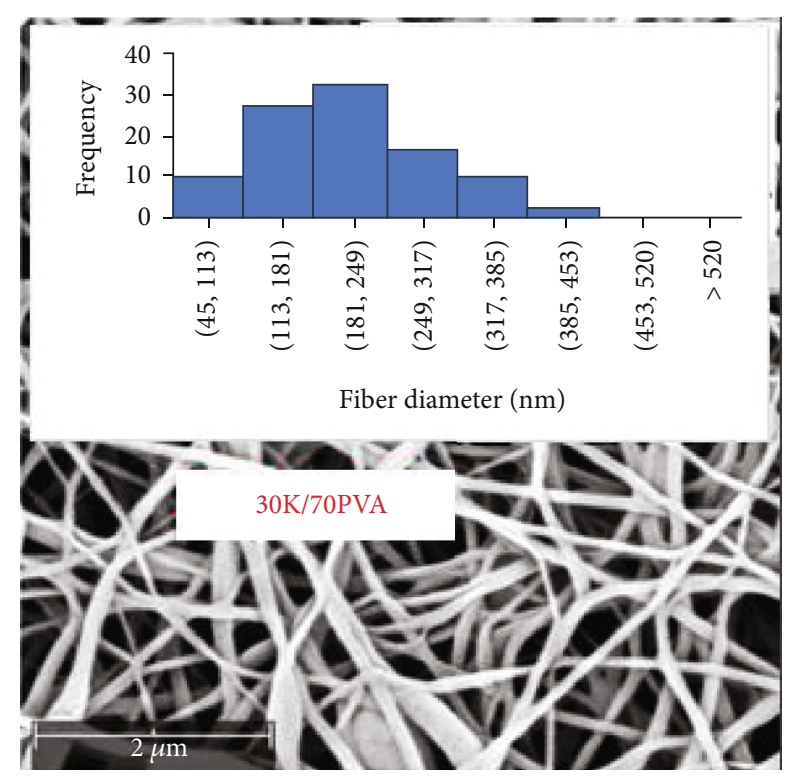

(d)

FIgURE 3: SEM images and distributions of nanofiber diameters of the keratin/polyvinyl alcohol system: (a) 0\% keratin/100\% PVA, (b) 10\% K/90\% PVA, (c) 20\% K/80\% PVA, and (d) 30\% K/70\% PVA.

support cellular ingrowth due to their ability to mimic the native tissue or organ $[13,14]$. Nanofibers can be electrospun into tubular forms that are flexible and soft to allow easy suturing of the distal and proximal stump during nerve repair. Furthermore, their porous nanostructure substrate for cell attachment allows nutrient exchange [5].

While biocompatible polymers have been used to fabricate electrospun nanofiber conduits [15], there is still a need for biodegradable polymers derived from natural resources to increase biocompatibility and lower immunological rejection. Among other synthetic polymers, polyvinyl alcohol (PVA) has been used in biomedical applications, including nerve repair treatment, due to its physical and chemical properties. Polyvinyl alcohol is FDA approved for clinical use in humans, and it has good electrospinnability to form nontoxic nanofibers with large pore sizes. However, cell adhesion for cell growth and biocompatibility need improvement $[16,17]$. Consequently, keratin has been used with PVA to increase scaffold biocompatibility. As a biodegradable fibrous protein, keratin can act as an extracellular matrix and promote biocompatibility to minimize immunological rejection. This protein has attracted interest from both scientific and industrial communities due to its exceptional properties and abundance. Interest includes investigating keratin application in the biomedical field. Feng et al. [18] developed keratin films to treat the corneal epithelial wound. It was then concluded that the keratin films were promising alternatives to the amniotic membrane for ocular 


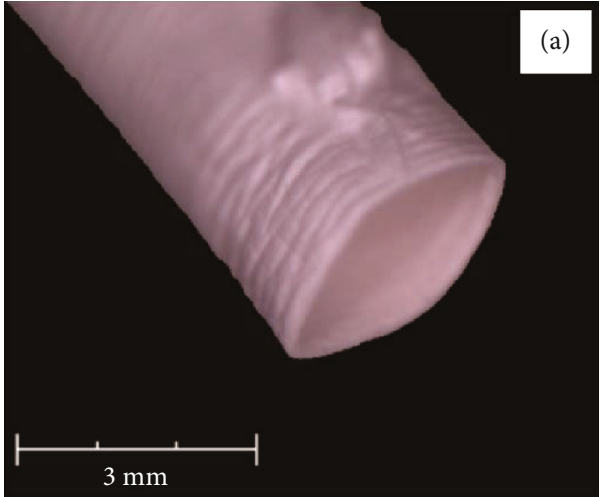

(a)

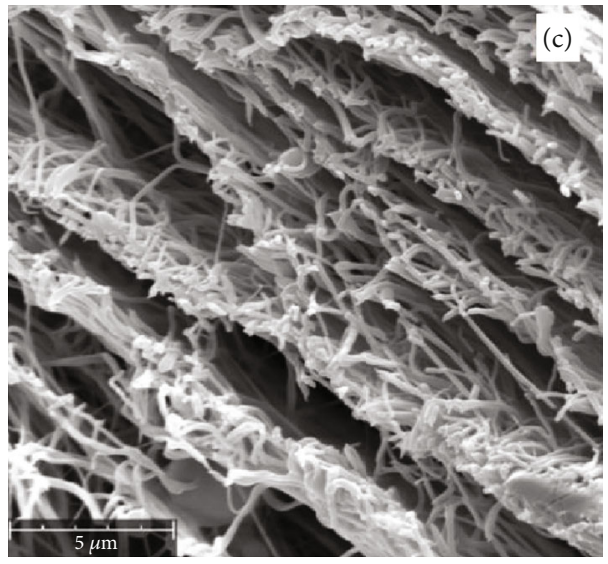

(c)

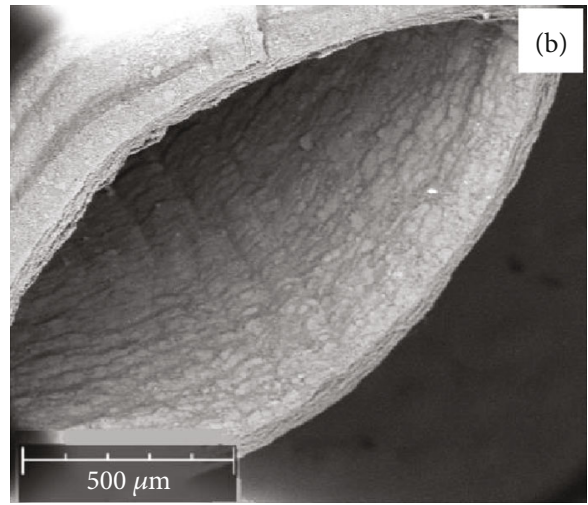

(b)

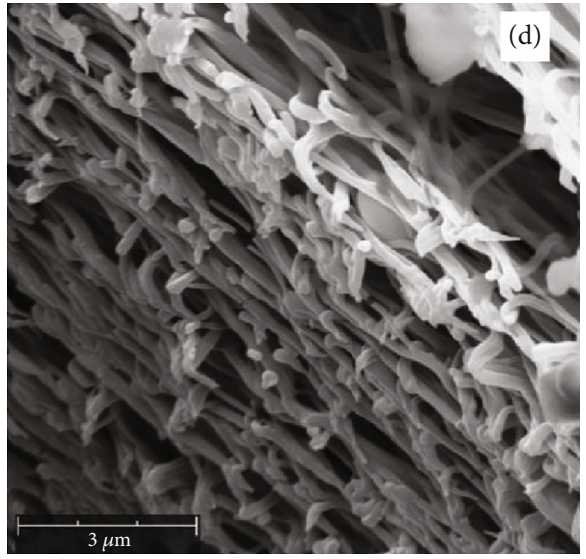

(d)

Figure 4: SEM images of keratin nanofibers: (a) tubular structure and (b) the outer and inner surfaces of the tube; (c, d) cross-sections of individual nanofibers, $0 \% \mathrm{~K} / 100 \%$ PVA and $10 \% \mathrm{~K} / 90 \%$ PVA nanofibers, respectively.

surface reconstruction; the films support proliferation, migration, adhesion, and differentiation of human corneal epithelial cells. The protein can also be used to exfoliate molybdenum disulphide $\left(\mathrm{MoS}_{2}\right)$ to achieve high-quality dispersion of nanolayered $\mathrm{MoS}_{2}$ with a high yield of about $56 \%$. The produced nanolayered sheet has a long shelf-life and improved electrical conductivity [19]. In another study, the effect of transglutaminase on wool keratin films showed a decrease in elongation at break and an increase in tensile strength, thus improving film stability while conserving drug release rate [20]. Further investigation by Ajay Sharma et al. [21] showed that keratin hydrogels could be used as a scaffold for pulp-dentine regeneration since keratin enhanced odontoblast cell behaviour. Choi et al. [22] developed keratin-based nanofibers with high optical transmittance of $88 \%$ at $600 \mathrm{~nm}$ with improved mechanical properties. Guo et al. [16] used oxidative hair keratin nanoparticles to coat PVA nanofibers for nerve repair, whereby large pore size enhanced neural cell viability and proliferation. Sierpinski et al. [23] produced a biomedical hair-keratin gel and concluded that it promotes vigorous nerve regeneration response by activating Schwann cells. Keratin inclusion facilitates the treatment result comparable to the gold standard conduit, the autograft. Similar results were observed with the application of hydrogel conduit filled with hair keratin for nerve regeneration, in which results were also comparable to the gold standard for nerve repair [24].

Therefore, the current study is aimed at fabricating and characterizing the seamless nanofiber conduit of polyvinyl alcohol and chicken feather keratin to apply in nerve regeneration.

\section{Methodology and Materials}

White chicken feathers were collected from the chicken meat processing plant RCL Foods at Hammarsdale, South Africa. Analytical grade sodium bisulphite, sodium hydroxide, sodium dodecyl sulphate, urea, and polyvinyl alcohol were purchased from Sigma-Aldrich, South Africa. The fabrication of seamless nanofiber conduits was done using Nano Spinner NE200 from Inovenso. The main instruments that were used for analysis were Fourier transform infrared spectroscopy (FTIR), Simultaneous Thermographic Analyzer (STA) 6000, and Carbon, Hydrogen, Nitrogen, Sulphur, and Oxygen (CHNS/O) Analyzer (Series II 2400) from PerkinElmer. Experiments, including keratin extraction, fabricating nanofibers and characterizations such as CHNS, were carried in triplicate, while the sample size of diameter measurements of nanofibers was 100 . 


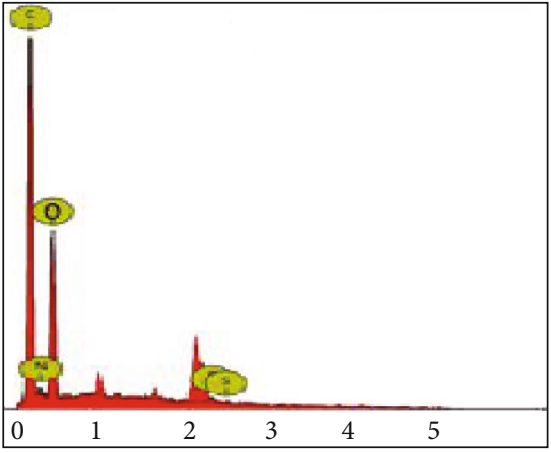

(a)

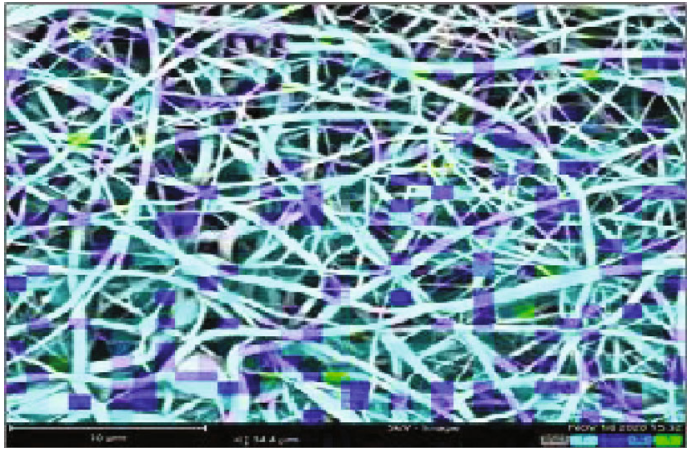

(b)

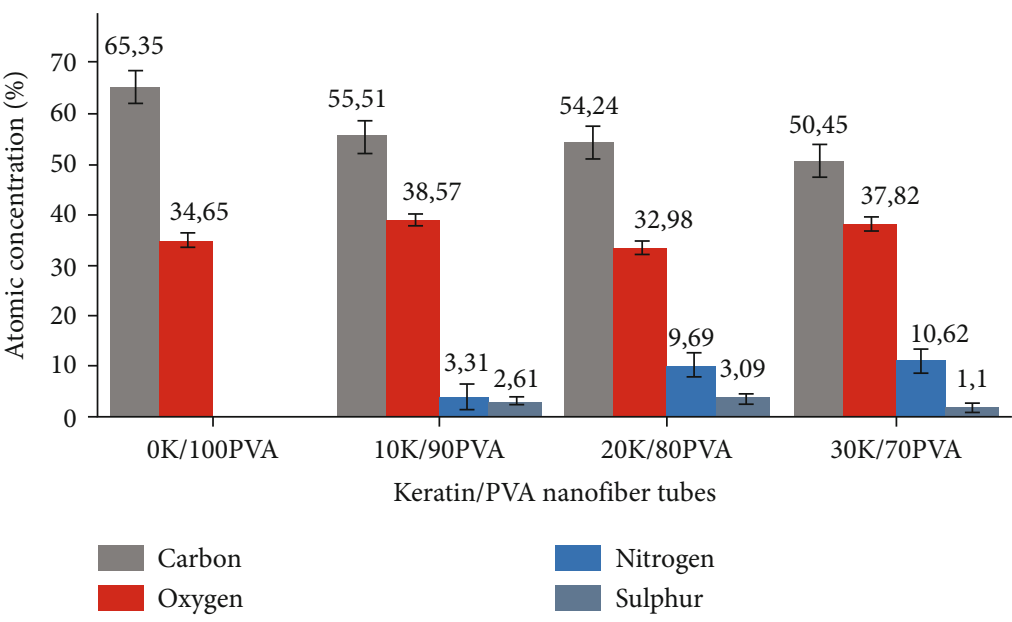

(c)

Figure 5: Energy-dispersive X-ray (EDX) results of keratin/PVA nanofiber tubes: (a) EDX spectrum of elements, (b) element mapping, and (c) atomic concentration of nanofibers.

TABLE 1: Conductivity of different keratin/PVA solutions and average diameters of relevant nanofibers.

\begin{tabular}{lccc}
\hline Keratin/PVA & Conductivity $(\mathrm{mS} / \mathrm{cm})$ & Average diameter \pm Std.dev $(\mathrm{nm})$ & Average pore size \pm Std.dev $(\mathrm{nm})$ \\
\hline $0 \% / 100 \%$ & 272 & $234 \pm 87$ & $0.3 \pm 0.081$ \\
$10 \% / 90 \%$ & 484 & $212 \pm 63$ & $0.09 \pm 0.029$ \\
$20 \% / 80 \%$ & 1020 & $196 \pm 37$ & $0.07 \pm 0.012$ \\
$30 \% / 70 \%$ & 1198 & $170 \pm 50$ & $0.04 \pm 0.004$ \\
\hline
\end{tabular}

2.1. Pretreatment of Chicken Feathers. Wet chicken feathers were rinsed with water at $60^{\circ} \mathrm{C}$ to remove excess blood while manually removing other meat by-products and then autoclaved at a temperature of $121^{\circ} \mathrm{C}$ and pressure $120 \mathrm{kPa}$ for 30 minutes. After that, they were soaked in $0.5 \% \mathrm{v} / v$ sodium hypochlorite for 24 hours before rinsing with water and dried at $25^{\circ} \mathrm{C}$.

2.2. Extraction and Analysis of Chicken Feather Keratin. Cleaned-disinfected chicken feathers were ground in a milling machine to increase the dissolution rate during the extraction process. They were then soaked in $99 \%$ ethanol for 24 hours to remove fatty materials and rinsed with water before drying at $50^{\circ} \mathrm{C}$ for three days. Fifteen grams of dry and degreased chicken feathers was deep in a solution of
0.23 M sodium bisulphite, $0.07 \mathrm{M}$ sodium dodecyl sulphate, and $1.5 \mathrm{M}$ urea. The reaction mixture was shaken on a linear platform shaker for homogeneous distribution of all mixture components in a container and then heated in a $90^{\circ} \mathrm{C}$ oil bath. After cooking, the mixture was centrifuged at $9000 \mathrm{rpm}$ for 15 minutes and filtered to separate the insoluble materials and supernatants. The filtrate obtained was dialyzed in distilled water using cellulose membrane dialysis tubes (MWCO $3.5 \mathrm{kDa}$ ) for five days. The keratin solution was lyophilized to obtain keratin powder, sealed, and stored in a cold room at $4^{\circ} \mathrm{C}$.

2.3. Preparation for Electrospinning Solutions. Solutions of various ratios of $0 / 100$ to $30 / 70$ of keratin/polyvinyl alcohol (K/PVA), at a constant concentration of $12 \mathrm{wt} \%$, were 


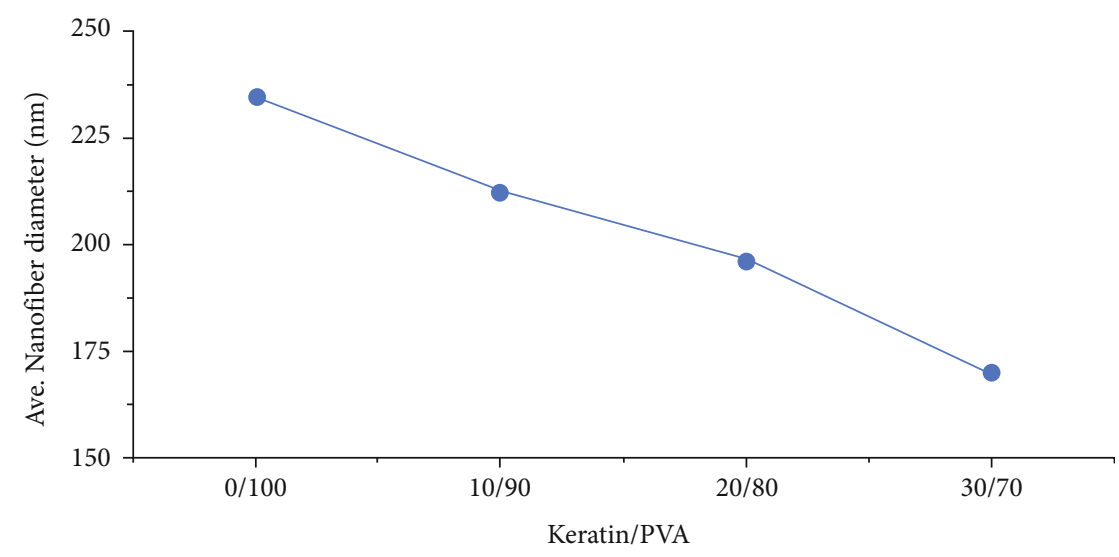

FIGURE 6: Graph of the effect of keratin content on average diameters of keratin/PVA nanofibers.
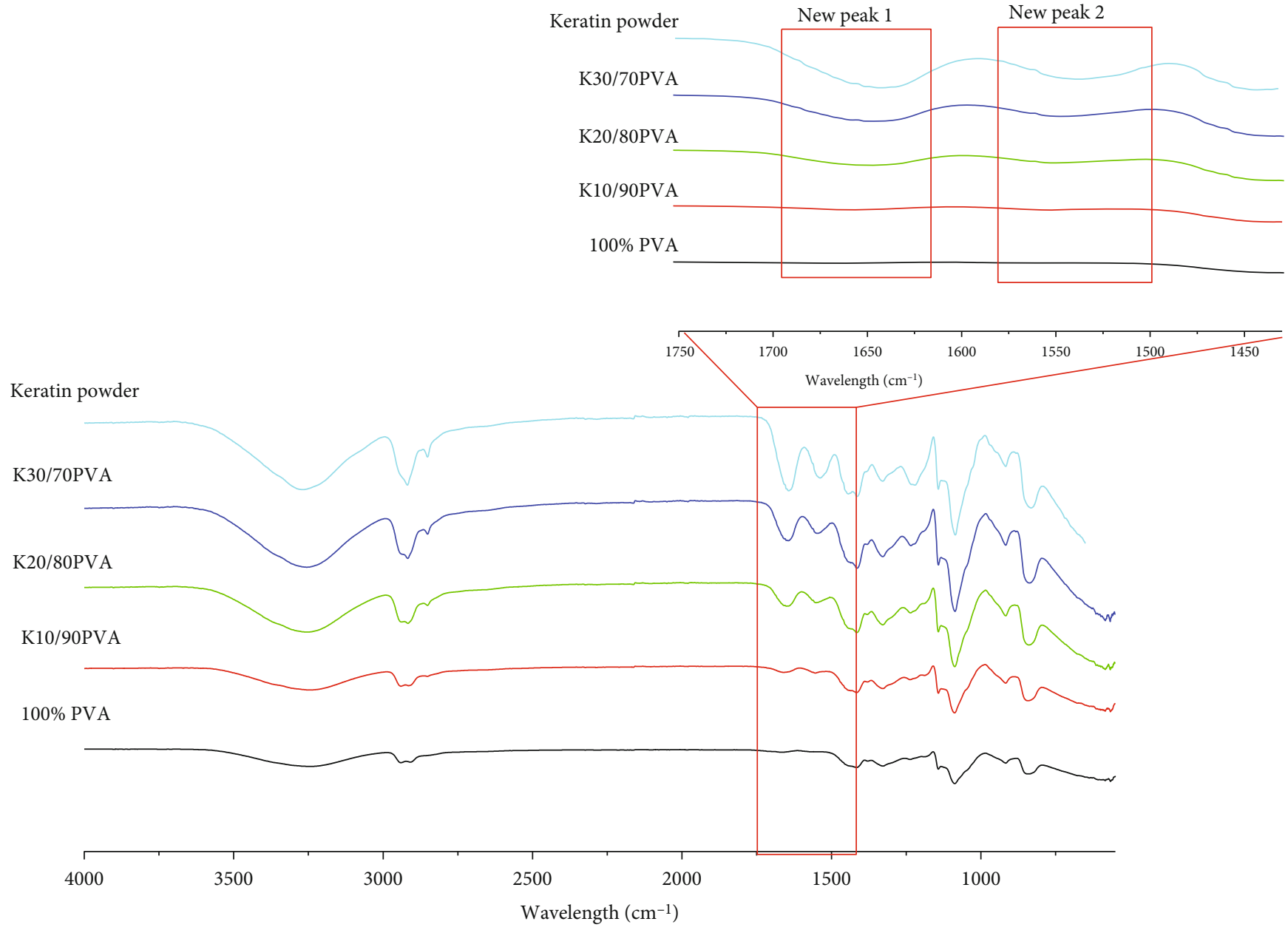

FIGURE 7: FTIR spectra of chicken feather keratin, PVA nanofibers, keratin/PVA nanofibers, and a zoomed-in subgraph of new peaks.

prepared by initially dissolving chicken feather keratin in deionized water, stirred at $50^{\circ} \mathrm{C}$ while adding about two drops of $1 \mathrm{M} \mathrm{NaOH}$, and then cooled to room temperature. Polyvinyl alcohol was slowly added into the solution while stirring and further stirred for 30 minutes; the temperature increased to $80^{\circ} \mathrm{C}$ for 2 hours before cooling to room temperature. The conductivity of the solutions was measured using Metrohm $914 \mathrm{pH} /$ conductometer, and the average of three measurements per solution was recorded. The solutions were then ready for electrospinning.

2.4. Fabrication of Seamless Nanofiber Conduits. Each electrospinning solution was placed in a $10 \mathrm{ml}$ polyurethane (PU) syringe; the syringe was then connected by a tube to 

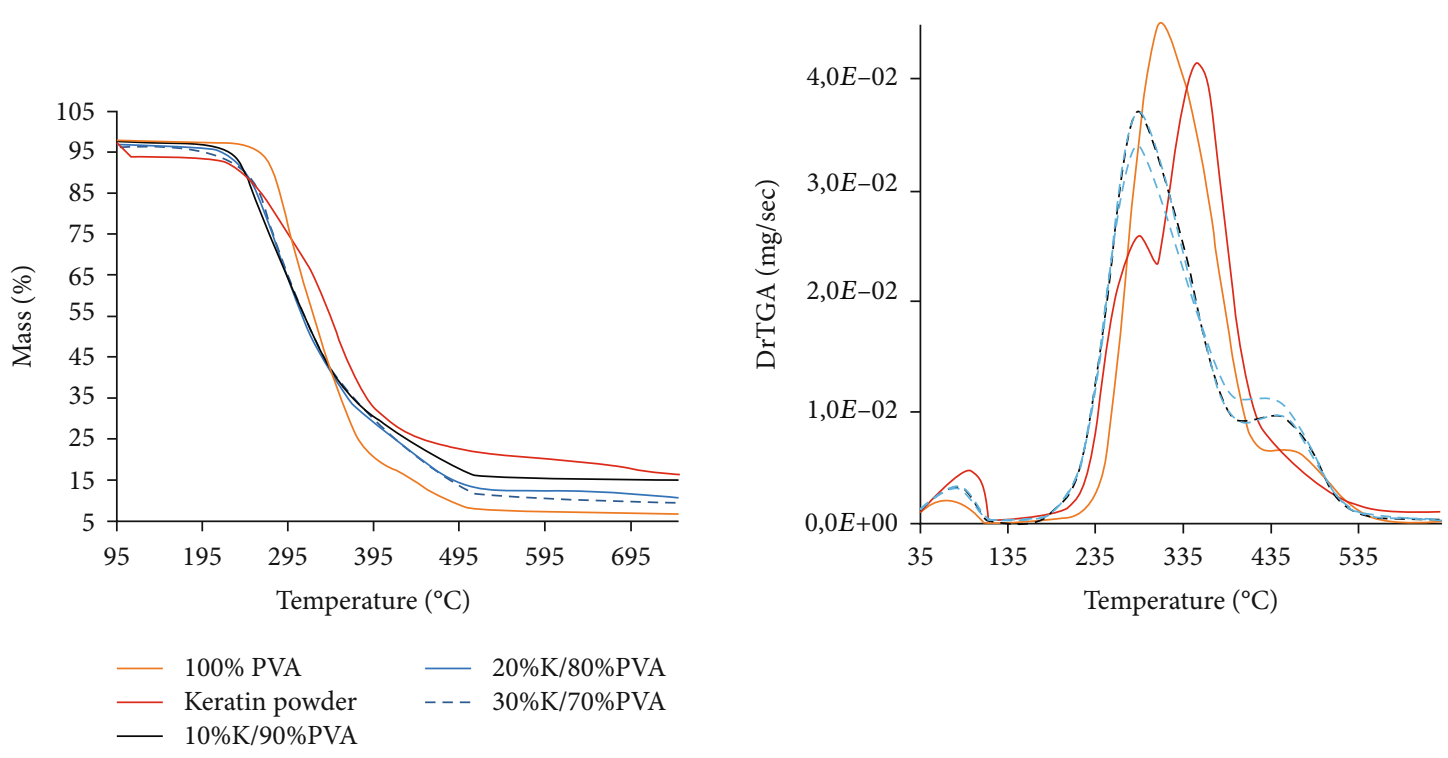

FIgURE 8: Thermogravimetric analysis and TGA derivative of keratin/PVA nanofibers.

TABle 2: Quantity of mass loss of keratin/PVA nanofibers at $100^{\circ} \mathrm{C}$ and $400^{\circ} \mathrm{C}$.

\begin{tabular}{lcc}
\hline & Mass loss (\%) & \\
\hline Sample & $T_{100 \circ \mathrm{C}}$ & $T_{400 \circ \mathrm{C}}$ \\
$100 \%$ PVA & 2.59 & 80.39 \\
$10 \%$ K/90\%P VA & 2.49 & 70.11 \\
$20 \%$ K/80\% PVA & 3.27 & 71.51 \\
$30 \%$ K/70\% PVA & 3.77 & 70.91 \\
Keratin powder & 3.98 & 68.11 \\
\hline
\end{tabular}

a nanospinner nozzle of $0.8 \mathrm{~mm}$ inside diameter. The electrospinning parameters were set to $30 \mathrm{kV}, 20 \mathrm{~cm}$ nozzlecollector distance, $3 \mathrm{ml} /$ hour feed rate, and a collector rotation speed of $52 \mathrm{~cm} / \mathrm{s}$.

\subsection{Characterization of Nanofiber Conduits}

2.5.1. Fourier Transform Infrared Spectroscopy (FTIR). Functional groups of the keratin, polyvinyl alcohol, and nanofibers were analyzed using Fourier transform infrared spectroscopy (Frontier Universal model, from PerkinElmer) in an attenuated total reflection mode (ATR). Each sample was scanned four times at a wavenumber range of 550$4000 \mathrm{~cm}^{-1}$ with a resolution of $4 \mathrm{~cm}^{-1}$.

2.5.2. CHNS Analysis for Crude Protein Content in Nanofibers. Protein content in nanofibers was done using a $\mathrm{CHNS/O}$ analyzer, which determines the content of carbon, hydrogen, nitrogen, and sulphur in the sample. After that, the protein content is calculated by multiplying the nitrogen content by a crude protein conversion factor of 6.25 [25].

2.5.3. Scanning Electron Microscopy (SEM). Scanning electron microscopy was used to determine morphological properties of nanofiber conduits such as shape, diameter, porosity, smoothness, and beads. Samples were set up on a metal stub using a sticky carbon disc; they were then goldcoated using a sputter coater before being placed in the ZEISS LEO 1450 Scanning Electron Microscope for imaging. Image-Pro Plus software was then used to analyze the diameters of 100 nanofibers.

2.5.4. Thermal Gravimetric Analysis. Thermophysical properties were investigated using a thermal gravimetric analyzer at a heating rate of $30^{\circ} \mathrm{C} / \mathrm{min}$ from 30 to $600^{\circ} \mathrm{C}$.

\section{Results and Discussions}

3.1. Extraction and Characterization of Keratin. White chicken feathers were obtained after pretreatment. The increased whiteness index of feathers was due to removing brownyellow oil and fats. The index further advanced after degreasing with ethanol. After that, a mixture of an aqueous solution and chicken feather particles was recovered after 4 hours of cooking. The final purification steps resulted in light brown supernatant and keratin powder after filtration and freeze-drying, respectively. Figure 1 contains the extracted keratin molecular weight's sodium dodecyl sulphate polyacrylamide gel electrophoresis (SDS-PAGE) analysis.

It shows that the extracted keratin has molecular weights that range from about $3 \mathrm{kDa}$ to approximately $60 \mathrm{kDa}$, whereas the broad, intense band between 10 and $15 \mathrm{kDa}$ indicates that multiple monomers have a molecular weight of $10-15 \mathrm{kDa}$ range [26].

FTIR's functional groups' qualitative analysis confirmed the presence of polypeptide amides, namely, amide A, amide $\mathrm{B}$, amide I, amide II, and amide III represented by the absorbance peaks at $3280.79 \mathrm{mc}^{-1}, 2919.88 \mathrm{mc}^{-1}, 1633.74 \mathrm{mc}^{-1}$, $1532.24 \mathrm{mc}^{-1}$, and $1212.05 \mathrm{mc}^{-1}$, respectively [27]. Furthermore, the extracted keratin elemental analysis showed that this keratin has $5.02 \%$ sulphur, $46.64 \%$ carbon, $10.82 \%$ nitrogen, $7.72 \%$ hydrogen, and $29.74 \%$ other elements, 
including oxygen. The extract is composed of $67.63 \%$ protein, calculated from the nitrogen percentage in the hydrolysate.

3.2. Preparation and Characterization of Keratin/PVA Nanofibers. The prepared electrospinning solutions of various ratios of keratin and PVA, from 00/100 to 30/70, were homogeneous and had a $\mathrm{pH}$ of 8 at $24.1^{\circ} \mathrm{C}$. The graph in Figure 2 shows the effect of keratin content in the electrospinning solution.

The conductivity of electrospinning solutions increased with an increase in keratin content. The increase in conductivity may be due to polar amino acids in keratin. The morphology of keratin/PVA nanofibers with different keratin contents from $0 \%$ to $30 \%$ is shown in Figure 3. All nanofibers were cylindrical and became thinner as keratin content was increased. As keratin content increases, the conductivity of the spinning solution increases.

The increase in conductivity increases the polymer jet's electrical charge, increases the electric field force, and results in thinner jets and nanofibers and smaller fiber diameters. In addition to greater electric field strength, increasing conductivity decreases the solution's viscosity, resulting in smaller nanofiber's average diameters. However, there was no noticeable change in the individual nanofibers' crosssectional shape from 100\%bPVA to 90\% PVA nanofibers, as shown in Figure 4.

Figure 3 also shows histograms of nanofiber diameters to indicate the diameter distributions that narrow as keratin content increases. Energy-dispersive X-ray (EDX) results, shown in Figure 5, indicate that keratin/PVA nanofibers are mainly composed of carbon, oxygen, nitrogen, and sulphur.

The element mapping of EDX showed that all the atoms were evenly distributed throughout the nanofibers' materials. The significant amounts of nitrogen and sulphur indicated the presence of keratin proteins. There was a noticeable increase in nitrogen percentage as keratin content increased in nanofibers. The conductivity and average diameters of nanofibers are tabulated in Table 1 and shown in Figure 6.

Furthermore, in Figure 5(b), SEM image analysis shows atoms evenly distributed throughout the nanofiber tubes. The average pore sizes decreased from 0.09 to $0.04 \mathrm{pm}$, decreasing with increased keratin content. This effect can also be attributed to the keratin conductivity effect; high conductivity within the polymer jet causes the jet to split into thinner jets due to the greater repulsive force, resulting in a higher number of fibers per unit area, thus reducing the number of fibers per unit pore size.

The FTIR spectra of PVA nanofibers, keratin powder, and keratin/PVA nanofibers are shown in Figure 7. Keratin spectrum was used as a reference spectrum for keratin/ PVA nanofiber spectra. The keratin spectrum exhibited peaks of different amides of keratin protein. A peak at $3282.56 \mathrm{~cm}^{-1}$ is $\mathrm{N}-\mathrm{H}$ stretching of amide $\mathrm{A}$ and possible Fermi resonance from the overtone of amide I [28], C-N bending of amide B $\left(2919.88 \mathrm{~cm}^{-1}\right)$, CO stretching of amide $\mathrm{I}\left(1633.74 \mathrm{~cm}^{-1}\right), \mathrm{C}-\mathrm{N}$ stretching and $\mathrm{N}-\mathrm{H}$ bending of amide
II $\left(1532.24 \mathrm{~cm}^{-1}\right)$, amide III's CO stretching $\left(1200 \mathrm{~cm}^{-1}\right)$, C$\mathrm{H}$ bending of amide IV $\left(623 \mathrm{~cm}^{-1}\right)$, and S-O stretching of cysteine-sulphonate $\left(1212.05 \mathrm{~cm}^{-1}\right)$ and disulphide bonds at $550 \mathrm{~cm}^{-1}[16,29]$. Some PVA spectrum peaks include -OH stretching peak at $3100 \mathrm{~cm}^{-1}$, -CHO stretching at $2950 \mathrm{~cm}^{-}$ ${ }^{1}$, and CO stretching at $2900 \mathrm{~cm}^{-1}$. The addition of keratin into PVA increased the intensity of amides $\mathrm{A}$ and $\mathrm{B}$; this can be attributed to the interaction of the $-\mathrm{NH}_{2}$ group of keratin and -OH group of PVA, resulting in hydrogen bond formation.

The increased amount of keratin corresponds with two new peaks related to PVA, at $1642.26 \mathrm{~cm}^{-1}$ and $1547.21 \mathrm{~cm}^{-}$ ${ }^{1}$. These peaks shift to shorter wavelengths as keratin content increases. The changes in the intensities of peaks, forming new ones and shifting to shorter wavelengths, confirm keratin in the nanofibers and suggest the chemical interactions between keratin and PVA. This chemical reaction between the PVA and keratin functional group minimizes the interaction between keratin macromolecules and increases the keratin's electrospinnability; the amide-carbonyl interaction further prevents phase separation of keratin and PVA in blended spinning solution [30].

TG analysis in Figure 8 shows the mass-loss curves and their derivatives to indicate polyvinyl alcohol nanofibers' thermal behaviour, keratin, and keratin-PVA nanofibers. These graphs show three major mass-loss events for nanofibers and keratin. The first event at just below $100^{\circ} \mathrm{C}$ was attributed to water evaporation. The mass loss, shown in Table 2, resulted in a mass reduction of $2.59 \%$ for $100 \%$ PVA nanofibers and $2.49 \%$ to $3.98 \%$ for $10 \%$ to $30 \%$ keratin nanofibers and keratin.

The increasing trend of water mass with the increase in PVA keratin content indicates keratin nanofibers' hydrophilicity. The second and significant mass loss occurred between $190^{\circ} \mathrm{C}$ and $400^{\circ} \mathrm{C}$. This was attributed to the degradation of alpha-helix and peptide bonds of amino acid residues [31].

The onset temperature of PVA nanofibers, $190^{\circ} \mathrm{C}$, was higher than that of keratin and keratin/PVA nanofibers at $160^{\circ} \mathrm{C}$. However, increasing keratin content showed no further effect on the onset temperature of keratin/PVA nanofibers. Nevertheless, PVA nanofibers and keratin showed a high degradation rate, with PVA nanofibers having the highest rate. Both PVA nanofibers and keratin exhibited a high degradation rate than their blend nanofibers. The third mass loss event occurred between $405^{\circ} \mathrm{C}$ and $490^{\circ} \mathrm{C}$; this loss was due to the degradation of the previous mass loss event's by-products. Keratin had a high amount of residuals than PVA, indicating better stability, while the ash content of keratin/PVA nanofibers fell between keratin and PVA.

\section{Conclusion}

Keratin/PVA nanofiber tubes were successfully fabricated without longitudinal seams. The addition of polyvinyl alcohol improved keratin's electrospinnability by interrupting keratin macromolecules' interaction and forming hydrogen bonds. On the other hand, keratin advanced the thermal stability of PVA nanofibers. Keratin nanofibers have a smaller 
diameter than keratin-free PVA nanofibers, increasing their surface area. The diameter of the nanofibers decreases when keratin content increases, suggesting the limit in electrospinnability of PVA/keratin blend due to keratin content. When used as nerve conduits, these permeable tubes will provide transportation of nutrients and metabolic waste; they will serve as a barrier that prevents other tissues into the regeneration area. Small diameters increase cell proliferation, cell spreading, and differentiation of neural stem cells while decreasing cell aggregation levels. The keratin-based nanofiber tubes are potential nerve regeneration frames as they mimic the extracellular matrix of the natural fibrous structure of neural tissue. They enhance electrophysiological recovery and axon density. Future investigations on the keratin/PVA nanofiber tubes will include in vitro and in vivo experiments.

\section{Data Availability}

The supporting data to the findings of this work will be available on request.

\section{Conflicts of Interest}

The authors declare that they have no conflicts of interest.

\section{Acknowledgments}

Acknowledgements for project funding and laboratory facilities are given to the Council for Scientific and Industrial Research-Biorefinery Industry Development Facility (CSIRBIDF), the Department of Science and Technology, and the University of KwaZulu-Natal. The first authors would also like to acknowledge Miss Nomthandazo Hadebe for helping with TGA.

\section{References}

[1] M. C. Dodla, M. Alvarado-Velez, V. J. Mukhatyar, and R. V. Bellamkonda, "Chapter 69- peripheral nerve regeneration," in Principles of Regenerative Medicine (Third Edition), A. Atala, R. Lanza, A. G. Mikos, and R. Nerem, Eds., pp. 12231236, Academic Press, Boston, 2019.

[2] J. Moskow, B. Ferrigno, N. Mistry et al., "Review: bioengineering approach for the repair and regeneration of peripheral nerve," Bioactive Materials, vol. 4, no. 1, pp. 107-113, 2019.

[3] X. Zhan, M. Gao, Y. Jiang et al., "Nanofiber scaffolds facilitate functional regeneration of peripheral nerve injury," Nanomedicine: Nanotechnology, Biology and Medicine, vol. 9, no. 3, pp. 305-315, 2013.

[4] N. Sinis, A. Kraus, D. Drakotos et al., "Bioartificial reconstruction of peripheral nerves using the rat median nerve model," Annals of Anatomy-Anatomischer Anzeiger, vol. 193, no. 4, pp. 341-346, 2011.

[5] S. Panseri, C. Cunha, J. Lowery et al., "Electrospun micro- and nanofiber tubes for functional nervous regeneration in sciatic nerve transections," BMC Biotechnology, vol. 8, no. 1, p. 39, 2008.

[6] R. Murphy, A. Faroni, J. Wong, and A. Reid, "Protocol for a phase I trial of a novel synthetic polymer nerve conduit 'Poly- nerve' in participants with sensory digital nerve injury (UMANC)," F1000Research, vol. 8, 2019.

[7] R. Tevlin, A. McArdle, D. Atashroo et al., "Biomaterials for craniofacial bone engineering," Journal of Dental Research, vol. 93, no. 12, pp. 1187-1195, 2014.

[8] J. Xue, T. Wu, Y. Dai, and Y. Xia, "Electrospinning and electrospun nanofibers: methods, materials, and applications," Chemical Reviews, vol. 119, no. 8, pp. 5298-5415, 2019.

[9] P. Zarrintaj, E. Zangene, S. Manouchehri et al., "Conductive biomaterials as nerve conduits: recent advances and future challenges," Applied Materials Today, vol. 20, article 100784, 2020.

[10] B. Sowmya, A. B. Hemavathi, and P. K. Panda, "Poly ( $\varepsilon$-caprolactone)-based electrospun nano-featured substrate for tissue engineering applications: a review," Progress in Biomaterials, vol. 10, no. 2, pp. 91-117, 2021.

[11] X. Zhang, W. Qu, D. Li et al., "Functional polymer-based nerve guide conduits to promote peripheral nerve regeneration," Advanced Materials Interfaces, vol. 7, no. 14, p. 2000225, 2020.

[12] S. L. Porter, S. M. Coulter, S. Pentlavalli, and G. Laverty, "Pharmaceutical formulation and characterization of dipeptide nanotubes for drug delivery applications," Macromolecular Bioscience, vol. 20, no. 7, p. 2000115, 2020.

[13] H. Yoshimoto, Y. M. Shin, H. Terai, and J. P. Vacanti, “A biodegradable nanofiber scaffold by electrospinning and its potential for bone tissue engineering," Biomaterials, vol. 24, no. 12, pp. 2077-2082, 2003.

[14] K. Kim and J. P. Fisher, "Nanoparticle technology in bone tissue engineering," Journal of Drug Targeting, vol. 15, no. 4, pp. 241-252, 2007.

[15] C. M. Agrawal and R. B. Ray, "Biodegradable polymeric scaffolds for musculoskeletal tissue engineering," Journal of Biomedical Materials Research, vol. 55, no. 2, pp. 141-150, 2001.

[16] T. Guo, X. Yang, J. Deng, L. Zhu, B. Wang, and S. Hao, "Keratin nanoparticles-coating electrospun PVA nanofibers for potential neural tissue applications," Journal of Materials Science: Materials in Medicine, vol. 30, no. 1, p. 9, 2018.

[17] X. Wang, T. Yucel, Q. Lu, X. Hu, and D. L. Kaplan, "Silk nanospheres and microspheres from silk/pva blend films for drug delivery," Biomaterials, vol. 31, no. 6, pp. 1025-1035, 2010.

[18] Y. Feng, M. Borrelli, T. Meyer-ter-Vehn, S. Reichl, S. Schrader, and G. Geerling, "Epithelial wound healing on keratin film, amniotic membrane and polystyrene in vitro," Current Eye Research, vol. 39, no. 6, pp. 561-570, 2014.

[19] V. Agarwal, N. Varghese, S. Dasgupta, A. K. Sood, and $\mathrm{K}$. Chatterjee, "Engineering a $3 \mathrm{D} \mathrm{MoS}_{2}$ foam using keratin exfoliated nanosheets," Chemical Engineering Journal, vol. 374, pp. 254-262, 2019.

[20] L. Cui, J. Gong, X. Fan, P. Wang, Q. Wang, and Y. Qiu, “Transglutaminase-modified wool keratin film and its potential application in tissue engineering," Engineering in Life Sciences, vol. 13, no. 2, pp. 149-155, 2013.

[21] L. Ajay Sharma, M. A. Ali, R. M. Love, M. J. Wilson, and G. J. Dias, "Novel keratin preparation supports growth and differentiation of odontoblast-like cells," International Endodontic Journal, vol. 49, no. 5, pp. 471-482, 2016.

[22] J. Choi, G. Panthi, Y. Liu et al., "Keratin/poly (vinyl alcohol) blended nanofibers with high optical transmittance," Polymer, vol. 58, pp. 146-152, 2015.

[23] P. Sierpinski, J. Garrett, J. Ma et al., "The use of keratin biomaterials derived from human hair for the promotion of rapid 
regeneration of peripheral nerves," Biomaterials, vol. 29, no. 1, pp. 118-128, 2008.

[24] L. A. Pace, J. F. Plate, T. L. Smith, and M. E. Van Dyke, “The effect of human hair keratin hydrogel on early cellular response to sciatic nerve injury in a rat model," Biomaterials, vol. 34, no. 24, pp. 5907-5914, 2013.

[25] P. P. Salo-väänänen and P. E. Koivistoinen, "Determination of protein in foods: comparison of net protein and crude protein $(\mathrm{N} \times 6.25)$ values," Food Chemistry, vol. 57, no. 1, pp. 27-31, 1996.

[26] P. Hill, H. Brantley, and M. Van Dyke, "Some properties of keratin biomaterials: kerateines," Biomaterials, vol. 31, no. 4, pp. 585-593, 2010.

[27] B. Ma, X. Qiao, X. Hou, and Y. Yang, "Pure keratin membrane and fibers from chicken feather," International Journal of Biological Macromolecules, vol. 89, pp. 614-621, 2016.

[28] V. Agarwal, A. G. Panicker, S. Indrakumar, and K. Chatterjee, "Comparative study of keratin extraction from human hair," International Journal of Biological Macromolecules, vol. 133, pp. 382-390, 2019.

[29] N. Ramakrishnan, S. Sharma, A. Gupta, and B. Y. Alashwal, "Keratin based bioplastic film from chicken feathers and its characterization," International Journal of Biological Macromolecules, vol. 111, pp. 352-358, 2018.

[30] I. Cruz-Maya, V. Guarino, A. Almaguer-Flores, M. A. AlvarezPerez, A. Varesano, and C. Vineis, "Highly polydisperse keratin rich nanofibers: scaffold design and in vitro characterization," Journal of Biomedical Materials Research Part A, vol. 107, no. 8, pp. 1803-1813, 2019.

[31] M. He, M. Chen, Y. Dou et al., "Electrospun silver nanoparticles-embedded feather keratin/poly (vinyl alcohol)/ poly(ethylene oxide) antibacterial composite nanofibers," Polymers, vol. 12, no. 2, p. 305, 2020. 